\title{
WEAK AND STRONG CONVERGENCE THEOREMS FOR RELATIVELY NONEXPANSIVE MAPPINGS IN BANACH SPACES
}

\author{
SHIN-YA MATSUSHITA AND WATARU TAKAHASHI
}

Received 29 October 2003

We first introduce an iterative sequence for finding fixed points of relatively nonexpansive mappings in Banach spaces, and then prove weak and strong convergence theorems by using the notion of generalized projection. We apply these results to the convex feasibility problem and a proximal-type algorithm for monotone operators in Banach spaces.

\section{Introduction}

Let $E$ be a real Banach space and let $A$ be a maximal monotone operator from $E$ to $E^{*}$, where $E^{*}$ is the dual space of $E$. It is well known that many problems in nonlinear analysis and optimization can be formulated as follows: find

$$
u \in E \quad \text { such that } 0 \in A u \text {. }
$$

A well-known method for solving (1.1) in a Hilbert space $H$ is the proximal point algorithm: $x_{0} \in H$ and

$$
x_{n+1}=J_{r_{n}} x_{n}, \quad n=0,1,2, \ldots,
$$

where $\left\{r_{n}\right\} \subset(0, \infty)$ and $J_{r}=(I+r A)^{-1}$ for all $r>0$. This algorithm was first introduced by Martinet [9]. In [16], Rockafellar proved that if $\liminf _{n \rightarrow \infty} r_{n}>0$ and $A^{-1} 0 \neq \varnothing$, then the sequence $\left\{x_{n}\right\}$ defined by (1.2) converges weakly to an element of solutions of (1.1). On the other hand, Kamimura and Takahashi [4] considered an algorithm to generate a strong convergent sequence in a Hilbert space. Further, Kamimura and Takahashi's result was extended to more general Banach spaces by Kohsaka and Takahashi [7]. They introduced and studied the following iteration sequence: $x=x_{0} \in E$ and

$$
x_{n+1}=J^{-1}\left(\alpha_{n} J x+\left(1-\alpha_{n}\right) J J_{r_{n}} x_{n}\right), \quad n=0,1,2, \ldots,
$$

where $J$ is the duality mapping on $E$ and $J_{r}=(J+r A)^{-1} J$ for all $r>0$. Kohsaka and Takahashi [7] proved that if $A^{-1} 0 \neq \varnothing, \lim _{n \rightarrow \infty} \alpha_{n}=0, \sum_{n=0}^{\infty} \alpha_{n}=\infty$, and $\lim _{n \rightarrow \infty} r_{n}=\infty$, then the sequence generated by (1.3) converges strongly to an element of $A^{-1} 0$. 
On the other hand, Reich [13] studied an iteration sequence of nonexpansive mappings in a Banach space which was first introduced by Mann [8]: $x_{0} \in C$ and

$$
x_{n+1}=\alpha_{n} x_{n}+\left(1-\alpha_{n}\right) S x_{n}, \quad n=0,1,2, \ldots,
$$

where $S$ is a nonexpansive mapping from a closed convex subset $C$ of $E$ into itself and $\left\{\alpha_{n}\right\} \subset[0,1]$. He proved that if $F(T)$ is nonempty and $\sum_{n=0}^{\infty} \alpha_{n}\left(1-\alpha_{n}\right)=\infty$, then the sequence generated by (1.4) converges weakly to some fixed point of $S$.

Motivated by Kohsaka and Takahashi [7], and Reich [13], our purpose in this paper is to prove weak and strong convergence theorems for relatively nonexpansive mappings in Banach spaces which were first introduced by Butnariu et al. [3] and further studied by the authors [10]. For this purpose, we consider the following iterative sequence: $x_{0} \in C$ and

$$
x_{n+1}=\Pi_{C} J^{-1}\left(\alpha_{n} J x_{n}+\left(1-\alpha_{n}\right) J T x_{n}\right), \quad n=0,1,2, \ldots,
$$

where $T$ is a relatively nonexpansive mapping from $C$ into itself and $\Pi_{C}$ is the generalized projection onto $C$. Notice that if $E$ is a Hilbert space and $S=T$, then the sequences (1.4) and (1.5) are equivalent. We prove that if $F(T)$ is nonempty and the duality mapping $J$ is weakly sequentially continuous, then the sequence $\left\{x_{n}\right\}$ converges weakly to a fixed point of $T$ and if the interior of $F(T)$ is nonempty, then $\left\{x_{n}\right\}$ converges strongly to a fixed point of $T$. Using these results, we also consider the convex feasibility problem and a proximal-type algorithm for monotone operators in Banach spaces.

\section{Preliminaries}

Let $E$ be a Banach space with norm $\|\cdot\|$ and let $E^{*}$ be the dual of $E$. Then we denote by $\left\langle x, x^{*}\right\rangle$ the pairing between $x \in E$ and $x^{*} \in E^{*}$. When $\left\{x_{n}\right\}$ is a sequence in $E$, we denote the strong convergence and the weak convergence of $\left\{x_{n}\right\}$ to $x \in E$ by $x_{n} \rightarrow x$ and $x_{n} \rightarrow x$, respectively.

A Banach space $E$ is said to be strictly convex if $\|(x+y) / 2\|<1$ for all $x, y \in E$ with $\|x\|=\|y\|=1$ and $x \neq y$. It is also said to be uniformly convex if $\lim _{n \rightarrow \infty}\left\|x_{n}-y_{n}\right\|=0$ for any two sequences $\left\{x_{n}\right\},\left\{y_{n}\right\}$ in $E$ such that $\left\|x_{n}\right\|=\left\|y_{n}\right\|=1$ and $\lim _{n \rightarrow \infty}\left\|\left(x_{n}+y_{n}\right) / 2\right\|=$ 1. The following result was proved by $\mathrm{Xu}[19]$.

Proposition 2.1 [19]. Let $r>0$ and let $E$ be a Banach space. If $E$ is uniformly convex, then there exists a continuous, strictly increasing, and convex function $g:[0, \infty) \rightarrow[0, \infty)$ with $g(0)=0$ such that

$$
\|\lambda x+(1-\lambda) y\|^{2} \leq \lambda\|x\|^{2}+(1-\lambda)\|y\|^{2}-\lambda(1-\lambda) g(\|x-y\|)
$$

for all $x, y \in B_{r}=\{z \in E:\|z\| \leq r\}$ and $\lambda$ with $0 \leq \lambda \leq 1$.

Let $U=\{x \in E:\|x\|=1\}$ be the unit sphere of $E$. The norm of $E$ is said to be Gâteaux differentiable if for each $x, y \in U$, the limit

$$
\lim _{t \rightarrow 0} \frac{\|x+t y\|-\|x\|}{t}
$$


exists. In this case, $E$ is called smooth. The norm of $E$ is said to be Fréchet differentiable if for each $x \in U$, the limit is attained uniformly for $y \in U$. It is also said to be uniformly smooth if the limit is attained uniformly for $x, y \in U$. The (normalized) duality mapping $J$ from $E$ to $E^{*}$ is defined by

$$
J x=\left\{x^{*} \in E^{*}:\left\langle x, x^{*}\right\rangle=\|x\|^{2}=\left\|x^{*}\right\|^{2}\right\}
$$

for $x \in E$. We say that $J$ is weakly sequentially continuous if for a sequence $\left\{x_{n}\right\} \subset E, x_{n} \rightarrow$ $x$, then $J x_{n} \stackrel{*}{\rightarrow} J x$, where $\stackrel{*}{\rightarrow}$ denotes the weak* convergence. We list several well-known properties of the duality mapping:

(1) if $E$ is smooth, then $J$ is single valued and norm-to-weak* continuous;

(2) if $E$ is Fréchet differentiable, then $J$ is norm-to-norm continuous;

(3) if $E$ is uniformly smooth, then $J$ is uniformly norm-to-norm continuous on each bounded subset of $E$.

For more details, see [17]. Assume that $E$ is smooth. Then the function $V: E \times E \rightarrow \mathbb{R}$ is defined by

$$
V(x, y)=\|x\|^{2}-2\langle x, J y\rangle+\|y\|^{2}
$$

for $x, y \in E$. From the definition of $V$, we have that

$$
(\|x\|-\|y\|)^{2} \leq V(x, y) \leq(\|x\|+\|y\|)^{2}
$$

for $x, y \in E$. The function $V$ also has the following property:

$$
V(y, x)=V(z, x)+V(y, z)+2\langle z-y, J x-J z\rangle
$$

for $x, y, z \in E$. The following result was proved by Kamimura and Takahashi [5].

Proposition 2.2 (Kamimura and Takahashi [5]). Let $r>0$ and let E be a uniformly convex and smooth Banach space. Then

$$
g(\|y-z\|) \leq V(y, z)
$$

for all $y, z \in B_{r}=\{w \in E:\|w\| \leq r\}$, where $g:[0, \infty) \rightarrow[0, \infty)$ is a continuous, strictly increasing, and convex function with $g(0)=0$.

Let $C$ be a nonempty closed convex subset of $E$. Suppose that $E$ is reflexive, strictly convex, and smooth. Then, for any $x \in E$, there exists a unique point $x_{0} \in C$ such that

$$
V\left(x_{0}, x\right)=\min _{y \in C} V(y, x)
$$

Following Alber [1], we denote such an $x_{0}$ by $\Pi_{C} x$. The mapping $\Pi_{C}$ is called the generalized projection from $E$ onto $C$. It is easy to see that in a Hilbert space, the mapping $\Pi_{C}$ is coincident with the metric projection. Concerning the generalized projection, the following are well known. 
Proposition 2.3 (Alber [1]; see also Kamimura and Takahashi [5]). Let C be a nonempty closed convex subset of a smooth Banach space $E$ and $x \in E$. Then

$$
x_{0}=\Pi_{C} x \Longleftrightarrow\left\langle x_{0}-y, J x-J x_{0}\right\rangle \geq 0 \quad \text { for each } y \in C .
$$

Proposition 2.4 (Alber [1]; see also Kamimura and Takahashi [5]). Let E be a reflexive, strictly convex, and smooth Banach space, let $C$ be a nonempty closed convex subset of $E$, and let $x \in E$. Then

$$
V\left(y, \Pi_{C} x\right)+V\left(\Pi_{C} x, x\right) \leq V(y, x) \quad \text { for each } y \in C
$$

Let $T$ be a mapping from $C$ into itself. We denote by $F(T)$ the set of fixed points of $T$. A point $p$ in $C$ is said to be an asymptotic fixed point of $T$ [12] if $C$ contains a sequence $\left\{x_{n}\right\}$ which converges weakly to $p$ such that the strong $\lim _{n \rightarrow \infty}\left(x_{n}-T x_{n}\right)=0$. The set of asymptotic fixed points of $T$ is denoted by $\hat{F}(T)$. We say that the mapping $T$ is called relatively nonexpansive $[3,10]$ if $F(T)=\hat{F}(T)$ and

$$
V(p, T x) \leq V(p, x) \quad \text { for each } x \in C, p \in F(T) .
$$

\section{Main results}

In this section, we discuss the weak and strong convergence of (1.5). To prove our results, we need the following proposition.

Proposition 3.1. Let E be a uniformly convex and smooth Banach space, let $C$ be a nonempty closed convex subset of $E$, and let $T$ be a relatively nonexpansive mapping from $C$ into itself such that $F(T)$ is nonempty. Let $\left\{\alpha_{n}\right\}$ be a sequence of real numbers such that $0 \leq \alpha_{n} \leq 1$. Suppose $\left\{x_{n}\right\}$ is the sequence generated by $x_{0} \in C$ and $x_{n+1}=\Pi_{C} J^{-1}\left(\alpha_{n} J x_{n}+\right.$ $\left.\left(1-\alpha_{n}\right) J T x_{n}\right), n=0,1,2, \ldots$ Then $\left\{\Pi_{F(T)} x_{n}\right\}$ converges strongly to some fixed point of $T$, where $\Pi_{F(T)}$ is the generalized projection from $C$ onto $F(T)$.

Proof. We know that $F(T)$ is closed and convex (see [10]). So, we can define the generalized projection $\Pi_{F(T)}$ onto $F(T)$. Let $p \in F(T)$. From Proposition 2.4 and the convexity of $\|\cdot\|^{2}$, we have

$$
\begin{aligned}
V\left(p, x_{n+1}\right) & =V\left(p, \Pi_{C} J^{-1}\left(\alpha_{n} J x_{n}+\left(1-\alpha_{n}\right) J T x_{n}\right)\right) \\
& \leq V\left(p, J^{-1}\left(\alpha_{n} J x_{n}+\left(1-\alpha_{n}\right) J T x_{n}\right)\right) \\
& =\|p\|^{2}-2\left\langle p, \alpha_{n} J x_{n}+\left(1-\alpha_{n}\right) J T x_{n}\right\rangle+\left\|\alpha_{n} J x_{n}+\left(1-\alpha_{n}\right) J T x_{n}\right\|^{2} \\
& \leq\|p\|^{2}-2 \alpha_{n}\left\langle p, J x_{n}\right\rangle-2\left(1-\alpha_{n}\right)\left\langle p, J T x_{n}\right\rangle+\alpha_{n}\left\|x_{n}\right\|^{2}+\left(1-\alpha_{n}\right)\left\|T x_{n}\right\|^{2} \\
& =\alpha_{n}\left(\|p\|^{2}-2\left\langle p, J x_{n}\right\rangle+\left\|x_{n}\right\|^{2}\right)+\left(1-\alpha_{n}\right)\left(\|p\|^{2}-2\left\langle p, J T x_{n}\right\rangle+\left\|T x_{n}\right\|^{2}\right) \\
& =\alpha_{n} V\left(p, x_{n}\right)+\left(1-\alpha_{n}\right) V\left(p, T x_{n}\right) \\
& \leq \alpha_{n} V\left(p, x_{n}\right)+\left(1-\alpha_{n}\right) V\left(p, x_{n}\right) \\
& =V\left(p, x_{n}\right) .
\end{aligned}
$$


Hence, $\lim _{n \rightarrow \infty} V\left(p, x_{n}\right)$ exists and, in particular, $\left\{V\left(p, x_{n}\right)\right\}$ is bounded. Then, by (2.5), $\left\{x_{n}\right\}$ is also bounded. This implies that $\left\{T x_{n}\right\}$ is bounded. Let $u_{n}=\Pi_{F(T)} x_{n}$ for each $n \in \mathbb{N} \cup\{0\}$. Then, we have

$$
V\left(u_{n}, x_{n+1}\right) \leq V\left(u_{n}, x_{n}\right)
$$

It follows from (2.10) that

$$
V\left(u_{n+1}, x_{n+1}\right)=V\left(\Pi_{F(T)} x_{n+1}, x_{n+1}\right) \leq V\left(u_{n}, x_{n+1}\right)-V\left(u_{n}, \Pi_{F(T)} x_{n+1}\right) .
$$

Combining this with (3.2), we obtain

$$
V\left(u_{n+1}, x_{n+1}\right) \leq V\left(u_{n}, x_{n}\right)
$$

It follows that $\left\{V\left(u_{n}, x_{n}\right)\right\}$ converges. Then, from (3.3),

$$
V\left(u_{n}, u_{n+1}\right) \leq V\left(u_{n}, x_{n}\right)-V\left(u_{n+1}, x_{n+1}\right)
$$

By induction, we have

$$
V\left(u_{n}, u_{n+m}\right) \leq V\left(u_{n}, x_{n}\right)-V\left(u_{n+m}, x_{n+m}\right)
$$

for each $m \in \mathbb{N}$. Using Proposition 2.2, we have, for $m, n$ with $n>m$,

$$
g\left(\left\|u_{m}-u_{n}\right\|\right) \leq V\left(u_{m}, u_{n}\right) \leq V\left(u_{m}, x_{m}\right)-V\left(u_{n}, x_{n}\right),
$$

where $g:[0, \infty) \rightarrow[0, \infty)$ is a continuous, strictly increasing, and convex function with $g(0)=0$. Then the properties of $g$ yield that $\left\{u_{n}\right\}$ is a Cauchy sequence. Since $E$ is complete and $F(T)$ is closed, $\left\{u_{n}\right\}$ converges strongly to some point $u$ in $F(T)$.

Now, we can prove a weak convergence theorem.

Theorem 3.2. Let E be a uniformly convex and uniformly smooth Banach space, let $C$ be a nonempty closed convex subset of $E$, let $T$ be a relatively nonexpansive mapping from $C$ into itself such that $F(T)$ is nonempty, and let $\left\{\alpha_{n}\right\}$ be a sequence of real numbers such that $0 \leq \alpha_{n} \leq 1$ and $\liminf _{n \rightarrow \infty} \alpha_{n}\left(1-\alpha_{n}\right)>0$. Suppose $\left\{x_{n}\right\}$ is the sequence generated by (1.5). If $J$ is weakly sequentially continuous, then $\left\{x_{n}\right\}$ converges weakly to some fixed point of $T$.

Proof. As in the proof of Proposition 3.1, we know that $\left\{x_{n}\right\}$ and $\left\{T x_{n}\right\}$ are bounded. Put $r=\sup _{n \in \mathbb{N} \cup\{0\}}\left\{\left\|x_{n}\right\|,\left\|T x_{n}\right\|\right\}$. Since $E$ is a uniformly smooth Banach space, $E^{*}$ is a uniformly convex Banach space (see $[17,18]$ for more details). Therefore, by Proposition 2.1, there exists a continuous, strictly increasing, and convex function $g:[0, \infty) \rightarrow[0, \infty)$ with $g(0)=0$ such that

$$
\left\|\lambda x^{*}+(1-\lambda) y^{*}\right\|^{2} \leq \lambda\left\|x^{*}\right\|^{2}+(1-\lambda)\left\|y^{*}\right\|^{2}-\lambda(1-\lambda) g\left(\left\|x^{*}-y^{*}\right\|\right)
$$


for each $x^{*}, y^{*} \in B_{r}=\left\{z^{*} \in E^{*}:\left\|z^{*}\right\| \leq r\right\}$ and $\lambda$ with $0 \leq \lambda \leq 1$. Let $p \in F(T)$. We have

$$
\begin{aligned}
V\left(p, x_{n+1}\right)= & V\left(p, \Pi_{C} J^{-1}\left(\alpha_{n} J x_{n}+\left(1-\alpha_{n}\right) J T x_{n}\right)\right) \\
\leq & V\left(p, J^{-1}\left(\alpha_{n} J x_{n}+\left(1-\alpha_{n}\right) J T x_{n}\right)\right) \\
= & \|p\|^{2}-2\left\langle p, \alpha_{n} J x_{n}+\left(1-\alpha_{n}\right) J T x_{n}\right\rangle+\left\|\alpha_{n} J x_{n}+\left(1-\alpha_{n}\right) J T x_{n}\right\|^{2} \\
\leq & \|p\|^{2}-2 \alpha_{n}\left\langle p, J x_{n}\right\rangle-2\left(1-\alpha_{n}\right)\left\langle p, J T x_{n}\right\rangle \\
& +\alpha_{n}\left\|x_{n}\right\|^{2}+\left(1-\alpha_{n}\right)\left\|T x_{n}\right\|^{2}-\alpha_{n}\left(1-\alpha_{n}\right) g\left(\left\|J x_{n}-J T x_{n}\right\|\right) \\
= & \alpha_{n} V\left(p, x_{n}\right)+\left(1-\alpha_{n}\right) V\left(p, T x_{n}\right)-\alpha_{n}\left(1-\alpha_{n}\right) g\left(\left\|J x_{n}-J T x_{n}\right\|\right) \\
\leq & V\left(p, x_{n}\right)-\alpha_{n}\left(1-\alpha_{n}\right) g\left(\left\|J x_{n}-J T x_{n}\right\|\right),
\end{aligned}
$$

and hence

$$
\alpha_{n}\left(1-\alpha_{n}\right) g\left(\left\|J x_{n}-J T x_{n}\right\|\right) \leq V\left(p, x_{n}\right)-V\left(p, x_{n+1}\right) .
$$

Since $\left\{V\left(p, x_{n}\right)\right\}$ converges and $\liminf _{n \rightarrow \infty} \alpha_{n}\left(1-\alpha_{n}\right)>0$, it follows that

$$
\lim _{n \rightarrow \infty} g\left(\left\|J x_{n}-J T x_{n}\right\|\right)=0 .
$$

Then the properties of $g$ yield that

$$
\lim _{n \rightarrow \infty}\left\|J x_{n}-J T x_{n}\right\|=0
$$

Since $J^{-1}$ is uniformly norm-to-norm continuous on bounded sets, we obtain

$$
\lim _{n \rightarrow \infty}\left\|x_{n}-T x_{n}\right\|=\lim _{n \rightarrow \infty}\left\|J^{-1}\left(J x_{n}\right)-J^{-1}\left(J T x_{n}\right)\right\|=0 .
$$

This implies that if there exists a subsequence $\left\{x_{n_{i}}\right\}$ of $\left\{x_{n}\right\}$ such that $x_{n_{i}} \rightarrow v$ for some $v \in E$, then, by the definition of $T, v$ is a fixed point of $T$.

Let $u_{n}=\Pi_{F(T)} x_{n}$ for each $n \in \mathbb{N} \cup\{0\}$. It follows from (2.9) that

$$
\left\langle u_{n}-z, J x_{n}-J u_{n}\right\rangle \geq 0
$$

for each $z \in F(T)$. Let $\left\{x_{n_{i}}\right\}$ be a subsequence of $\left\{x_{n}\right\}$ such that $\left\{x_{n_{i}}\right\}$ converges weakly to $v$. Then we have $v \in F(T)$. By (3.14), we have

$$
\left\langle u_{n_{i}}-v, J x_{n_{i}}-J u_{n_{i}}\right\rangle \geq 0 .
$$

From Proposition 3.1, we know that $\left\{u_{n}\right\}$ converges strongly to some $u \in F(T)$ and $J$ is weakly sequentially continuous. Letting $i \rightarrow \infty$, we have

$$
\langle u-v, J v-J u\rangle \geq 0
$$

On the other hand, from the monotonicity of $J$, we have

$$
\langle u-v, J u-J v\rangle \geq 0
$$


Combining this with (3.16), we have

$$
\langle u-v, J u-J v\rangle=0
$$

Using the strict convexity of $E$, we obtain $u=v$. Therefore, $\left\{x_{n}\right\}$ converges weakly to $u=\lim _{n \rightarrow \infty} \Pi_{F(T)} x_{n}$. This completes the proof.

Next, we also consider the strong convergence of (1.5). We can prove the following theorem without the assumption of "weakly sequentially continuous" in the duality mapping $J$.

Theorem 3.3. Let E be a uniformly convex and uniformly smooth Banach space, let $C$ be a nonempty closed convex subset of $E$, let $T$ be a relatively nonexpansive mapping from $C$ into itself, and let $\left\{\alpha_{n}\right\}$ be a sequence of real numbers such that $0 \leq \alpha_{n} \leq 1$ and $\liminf _{n \rightarrow \infty} \alpha_{n}(1-$ $\left.\alpha_{n}\right)>0$. Suppose $\left\{x_{n}\right\}$ is the sequence generated by (1.5). If the interior of $F(T)$ is nonempty, then $\left\{x_{n}\right\}$ converges strongly to some fixed point of $T$.

Proof. Since the interior of $F(T)$ is nonempty, there exist $p \in F(T)$ and $r>0$ such that

$$
p+r h \in F(T)
$$

whenever $\|h\| \leq 1$. By (2.6), we have, for any $u \in F(T)$,

$$
V\left(u, x_{n}\right)=V\left(x_{n+1}, x_{n}\right)+V\left(u, x_{n+1}\right)+2\left\langle x_{n+1}-u, J x_{n}-J x_{n+1}\right\rangle .
$$

This implies

$$
\left\langle x_{n+1}-u, J x_{n}-J x_{n+1}\right\rangle+\frac{1}{2} V\left(x_{n+1}, x_{n}\right)=\frac{1}{2}\left(V\left(u, x_{n}\right)-V\left(u, x_{n+1}\right)\right) .
$$

We also have

$$
\begin{aligned}
\left\langle x_{n+1}-p, J x_{n}-J x_{n+1}\right\rangle & =\left\langle x_{n+1}-(p+r h)+r h, J x_{n}-J x_{n+1}\right\rangle \\
& =\left\langle x_{n+1}-(p+r h), J x_{n}-J x_{n+1}\right\rangle+r\left\langle h, J x_{n}-J x_{n+1}\right\rangle .
\end{aligned}
$$

On the other hand, since $p+r h \in F(T)$, as in the proof of Proposition 3.1, we have that

$$
V\left(p+r h, x_{n+1}\right) \leq V\left(p+r h, x_{n}\right) .
$$

From (3.21), this inequality is equivalent to

$$
0 \leq\left\langle x_{n+1}-(p+r h), J x_{n}-J x_{n+1}\right\rangle+\frac{1}{2} V\left(x_{n+1}, x_{n}\right) .
$$

Then, by (3.21), we have

$$
\begin{aligned}
r\left\langle h, J x_{n}-J x_{n+1}\right\rangle & \leq\left\langle x_{n+1}-p, J x_{n}-J x_{n+1}\right\rangle+\frac{1}{2} V\left(x_{n+1}, x_{n}\right) \\
& =\frac{1}{2}\left(V\left(p, x_{n}\right)-V\left(p, x_{n+1}\right)\right),
\end{aligned}
$$


and hence

$$
\left\langle h, J x_{n}-J x_{n+1}\right\rangle \leq \frac{1}{2 r}\left(V\left(p, x_{n}\right)-V\left(p, x_{n+1}\right)\right) .
$$

Since $h$ with $\|h\| \leq 1$ is arbitrary, we have

$$
\left\|J x_{n}-J x_{n+1}\right\| \leq \frac{1}{2 r}\left(V\left(p, x_{n}\right)-V\left(p, x_{n+1}\right)\right) .
$$

So, if $n>m$, then

$$
\begin{aligned}
\left\|J x_{m}-J x_{n}\right\| & =\left\|J x_{m}-J x_{m+1}+J x_{m+1}-\cdots-J x_{n-1}+J x_{n-1}-J x_{n}\right\| \\
& \leq \sum_{i=m}^{n-1}\left\|J x_{i}-J x_{i+1}\right\| \leq \frac{1}{2 r} \sum_{i=m}^{n-1}\left(V\left(p, x_{i}\right)-V\left(p, x_{i+1}\right)\right) \\
& =\frac{1}{2 r}\left(V\left(p, x_{m}\right)-V\left(p, x_{n}\right)\right) .
\end{aligned}
$$

We know that $\left\{V\left(p, x_{n}\right)\right\}$ converges. So, $\left\{J x_{n}\right\}$ is a Cauchy sequence. Since $E^{*}$ is complete, $\left\{J x_{n}\right\}$ converges strongly to some point in $E^{*}$. Since $E^{*}$ has a Fréchet differentiable norm, then $J^{-1}$ is continuous on $E^{*}$. Hence, $\left\{x_{n}\right\}$ converges strongly to some point $u$ in $C$. As in the proof of Theorem 3.2, we also have that $\left\|x_{n}-T x_{n}\right\| \rightarrow 0$. So, we have $u \in F(T)$, where $u=\lim _{n \rightarrow \infty} \Pi_{F(T)} x_{n}$.

\section{Applications}

In this section, using Theorems 3.2 and 3.3, we give some applications. We first consider the problem of weak convergence concerning nonexpansive mappings in a Hilbert space.

Theorem 4.1 (Browder and Petryshyn [2]). Let $C$ be a nonempty closed convex subset of a Hilbert space $H$, let $T$ be a nonexpansive mapping from $C$ into itself such that $F(T)$ is nonempty, and let $\lambda$ be a real number such that $0<\lambda<1$. Suppose that $\left\{x_{n}\right\}$ is given by $x_{0} \in C$ and

$$
x_{n+1}=\lambda x_{n}+(1-\lambda) T x_{n}, \quad n=0,1,2, \ldots
$$

Then $\left\{x_{n}\right\}$ converges weakly to $u$ in $F(T)$, where $u=\lim _{n \rightarrow \infty} P_{F(T)} x_{n}$ and $P_{F(T)}$ is the metric projection from $C$ onto $F(T)$.

Proof. Let $\alpha_{n}=\lambda$ for each $n \in \mathbb{N} \cup\{0\}$. It is clear that $\liminf _{n \rightarrow \infty} \alpha_{n}\left(1-\alpha_{n}\right)=\lambda(1-\lambda)>0$. We show that if $T$ is nonexpansive, then $T$ is relatively nonexpansive. It is obvious that $F(T) \subset \hat{F}(T)$. If $u \in \hat{F}(T)$, then there exists $\left\{x_{n}\right\} \subset C$ such that $x_{n} \rightarrow u$ and $x_{n}-T x_{n} \rightarrow 0$. Since $T$ is nonexpansive, $T$ is demiclosed. So, we have $u=T u$. This implies $F(T)=\hat{F}(T)$. Further, in a Hilbert space $H$, we know that

$$
V(x, y)=\|x-y\|^{2}
$$

for every $x, y \in H$. So, $\|T x-T y\| \leq\|x-y\|$ is equivalent to $V(T x, T y) \leq V(x, y)$. Therefore, $T$ is relatively nonexpansive. Using Theorem 3.2, we obtain the desired result. 
We also consider the strong convergence concerning nonexpansive mappings in a Hilbert space. For related results, see Moreau [11], and Kirk and Sims [6].

Theorem 4.2. Let $C$ be a nonempty closed convex subset of a Hilbert space $H$, let $T$ be a nonexpansive mapping from $C$ into itself, and let $\lambda$ be a real number such that $0<\lambda<1$. Suppose that $\left\{x_{n}\right\}$ is given by $x_{0} \in C$ and

$$
x_{n+1}=\lambda x_{n}+(1-\lambda) T x_{n}, \quad n=0,1,2, \ldots
$$

If the interior of $F(T)$ is nonempty, then $\left\{x_{n}\right\}$ converges strongly to $u$ in $F(T)$, where $u=$ $\lim _{n \rightarrow \infty} P_{F(T)} x_{n}$ and $P_{F(T)}$ is the metric projection from $C$ onto $F(T)$.

Next, we apply Theorems 3.2 and 3.3 to the convex feasibility problem. Before giving them, we introduce the following lemma which was proved by Reich [12].

LeMma 4.3 (Reich [12]). Let E be a uniformly convex Banach space with a uniformly Gâteaux-differentiable norm, let $\left\{C_{i}\right\}_{i=1}^{m}$ be a finite family of closed convex subsets of $E$, and let $\Pi_{i}$ be the generalized projection from $E$ onto $C_{i}$ for each $i=1,2, \ldots, m$. Then

$$
V\left(p, \Pi_{m} \Pi_{m-1} \cdots \Pi_{2} \Pi_{1} x\right) \leq V(p, x)
$$

for each $p \in \hat{F}\left(\Pi_{m} \Pi_{m-1} \cdots \Pi_{2} \Pi_{1}\right), x \in E$, and $\hat{F}\left(\Pi_{m} \Pi_{m-1} \cdots \Pi_{2} \Pi_{1}\right)=\cap_{i=1}^{m} C_{i}$.

As direct consequences of Lemma 4.3 and Theorems 3.2 and 3.3, we can prove the following two results.

THeORem 4.4. Let E be a uniformly convex and uniformly smooth Banach space, let $\left\{C_{i}\right\}_{i=1}^{m}$ be a finite family of closed convex subsets of $E$ such that $\cap_{i=1}^{m} C_{i}$ is nonempty, let $\Pi_{i}$ be the generalized projection from $E$ onto $C_{i}$ for each $i=1,2, \ldots, m$, and let $\left\{\alpha_{n}\right\}$ be a sequence of real numbers such that $0 \leq \alpha_{n} \leq 1$ and $\liminf _{n \rightarrow \infty} \alpha_{n}\left(1-\alpha_{n}\right)>0$. Suppose that $\left\{x_{n}\right\}$ is given by $x_{0} \in E$ and

$$
x_{n+1}=J^{-1}\left(\alpha_{n} J x_{n}+\left(1-\alpha_{n}\right) J \Pi_{m} \Pi_{m-1} \cdots \Pi_{2} \Pi_{1} x_{n}\right), \quad n=0,1,2, \ldots
$$

If $J$ is weakly sequentially continuous, then $\left\{x_{n}\right\}$ converges weakly to $u$ in $\cap_{i=1}^{m} C_{i}$, where $u=\lim _{n \rightarrow \infty} \prod_{\cap_{i=1}^{m} C_{i}} x_{n}$ and $\Pi_{\cap_{i=1}^{m} C_{i}}$ is the generalized projection from E onto $\cap_{i=1}^{m} C_{i}$.

Proof. Put $T=\Pi_{m} \Pi_{m-1} \cdots \Pi_{2} \Pi_{1}$. It is clear that $F(T) \subset \hat{F}(T)$ and $\cap_{i=1}^{m} C_{i} \subset F(T)$. By Lemma 4.3, we have that $T$ is a relatively nonexpansive mapping and $F(T)=\cap_{i=1}^{m} C_{i}$. Applying Theorem 3.2, $\left\{x_{n}\right\}$ converges weakly to $u=\lim _{n \rightarrow \infty} \prod_{\cap_{i=1}^{m} C_{i}} x_{n}$.

THeorem 4.5. Let E be a uniformly convex and uniformly smooth Banach space, let $\left\{C_{i}\right\}_{i=1}^{m}$ be a finite family of closed convex subsets of $E$, let $\Pi_{i}$ be the generalized projection from $E$ onto $C_{i}$ for each $i=1,2, \ldots, m$, and let $\left\{\alpha_{n}\right\}$ be a sequence of real numbers such that $0 \leq \alpha_{n} \leq 1$ and $\liminf _{n \rightarrow \infty} \alpha_{n}\left(1-\alpha_{n}\right)>0$. Suppose that $\left\{x_{n}\right\}$ is given by $x_{0} \in E$ and

$$
x_{n+1}=J^{-1}\left(\alpha_{n} J x_{n}+\left(1-\alpha_{n}\right) J \Pi_{m} \Pi_{m-1} \cdots \Pi_{2} \Pi_{1} x_{n}\right), \quad n=0,1,2, \ldots
$$

If the interior of $\cap_{i=1}^{m} C_{i}$ is nonempty, then $\left\{x_{n}\right\}$ converges strongly to $u$ in $\cap_{i=1}^{m} C_{i}$, where $u=\lim _{n \rightarrow \infty} \prod_{\cap_{i=1}^{m} C_{i}} x_{n}$ and $\prod_{\cap_{i=1}^{m} C_{i}}$ is the generalized projection from $E$ onto $\cap_{i=1}^{m} C_{i}$. 
Let $A$ be a multivalued operator with the domain $D(A)=\{x \in E: A x \neq \varnothing\}$ and the graph $G(A)=\left\{\left(x, x^{*}\right) \in E \times E^{*}: x^{*} \in A x\right\}$. The operator $A$ is said to be monotone if

$$
\left\langle x-y, x^{*}-y^{*}\right\rangle \geq 0 \text { for each }\left(x, x^{*}\right),\left(y, y^{*}\right) \in G(A) .
$$

The operator $A$ is maximal monotone if $A$ is monotone, and for any monotone operator $B$ from $E$ to $E^{*}$ with $G(A) \subset G(B)$, we have $A=B$. We know that if $A$ is maximal monotone, then $A^{-1} 0$ is closed and convex. The following result is also well known.

Theorem 4.6 (Rockafellar [15]). Let E be a reflexive, strictly convex, and smooth Banach space and let $A$ be a monotone operator from $E$ to $E^{*}$. Then $A$ is maximal if and only if $R(J+r A)=E^{*}$ for all $r>0$.

Let $E$ be a reflexive, strictly convex, and smooth Banach space and let $A$ be a maximal monotone operator from $E$ to $E^{*}$. Using Theorem 4.6 and the strict convexity of $E$, we obtain that for every $r>0$ and $x \in E$, there exists a unique $x_{r} \in D(A)$ such that

$$
J x \in J x_{r}+r A x_{r} .
$$

If $J_{r} x=x_{r}$, then we can define a single-valued mapping $J_{r}: E \rightarrow D(A)$ by $J_{r}=(J+r A)^{-1} J$. Such a $J_{r}$ is called the resolvent of $A$. We know that $J_{r}$ is relatively nonexpansive (see $[10,12,14]$ ), and $A^{-1} 0=F\left(J_{r}\right)$ for all $r>0$ (see $[17,18]$ ). As direct consequences of Theorems 3.2 and 3.3, we also have the following two results.

Theorem 4.7. Let E be a uniformly convex and uniformly smooth Banach space, let $A$ be a maximal monotone operator from $E$ to $E^{*}$ such that $A^{-1} 0$ is nonempty, let $J_{r}$ be the resolvent of $A$, where $r>0$, and let $\left\{\alpha_{n}\right\}$ be a sequence of real numbers such that $0 \leq \alpha_{n} \leq 1$ and $\liminf _{n \rightarrow \infty} \alpha_{n}\left(1-\alpha_{n}\right)>0$. Suppose the sequence $\left\{x_{n}\right\}$ is given by $x_{0} \in E$ and

$$
x_{n+1}=J^{-1}\left(\alpha_{n} J x_{n}+\left(1-\alpha_{n}\right) J J_{r} x_{n}\right), \quad n=0,1,2, \ldots
$$

If $J$ is weakly sequentially continuous, then $\left\{x_{n}\right\}$ converges weakly to $u$ in $A^{-1} 0$, where $u=$ $\lim _{n \rightarrow \infty} \Pi_{A^{-1} 0} x_{n}$ and $\Pi_{A^{-1} 0}$ is the generalized projection from $E$ onto $A^{-1} 0$.

Theorem 4.8. Let E be a uniformly convex and uniformly smooth Banach space, let $A$ be a maximal monotone operator from $E$ to $E^{*}$, let $J_{r}$ be the resolvent of $A$, where $r>0$, and let $\left\{\alpha_{n}\right\}$ be a sequence of real numbers such that $0 \leq \alpha_{n} \leq 1$ and $\liminf _{n \rightarrow \infty} \alpha_{n}\left(1-\alpha_{n}\right)>0$. Suppose the sequence $\left\{x_{n}\right\}$ is given by $x_{0} \in E$ and

$$
x_{n+1}=J^{-1}\left(\alpha_{n} J x_{n}+\left(1-\alpha_{n}\right) J J_{r} x_{n}\right), \quad n=0,1,2, \ldots
$$

If the interior of $A^{-1} 0$ is nonempty, then $\left\{x_{n}\right\}$ converges strongly to $u$ in $A^{-1} 0$, where $u=$ $\lim _{n \rightarrow \infty} \Pi_{A^{-1} 0} x_{n}$ and $\Pi_{A^{-1} 0}$ is the generalized projection from $E$ onto $A^{-1} 0$.

\section{References}

[1] Y. I. Alber, Metric and generalized projection operators in Banach spaces: properties and applications, Theory and Applications of Nonlinear Operators of Accretive and Monotone Type (A. G. Kartsatos, ed.), Lecture Notes in Pure and Appl. Math., vol. 178, Marcel Dekker, New York, 1996, pp. 15-50. 
[2] F. E. Browder and W. V. Petryshyn, Construction of fixed points of nonlinear mappings in Hilbert space, J. Math. Anal. Appl. 20 (1967), 197-228.

[3] D. Butnariu, S. Reich, and A. J. Zaslavski, Asymptotic behavior of relatively nonexpansive operators in Banach spaces, J. Appl. Anal. 7 (2001), no. 2, 151-174.

[4] S. Kamimura and W. Takahashi, Approximating solutions of maximal monotone operators in Hilbert spaces, J. Approx. Theory 106 (2000), no. 2, 226-240.

[5] - Strong convergence of a proximal-type algorithm in a Banach space, SIAM J. Optim. 13 (2002), no. 3, 938-945.

[6] W. A. Kirk and B. Sims, Convergence of Picard iterates of nonexpansive mappings, Bull. Polish Acad. Sci. Math. 47 (1999), no. 2, 147-155.

[7] F. Kohsaka and W. Takahashi, Strong convergence of an iterative sequence for maximal monotone operators in a Banach space, to appear in Abstr. Appl. Anal.

[8] W. R. Mann, Mean value methods in iteration, Proc. Amer. Math. Soc. 4 (1953), 506-510.

[9] B. Martinet, Régularisation d'inéquations variationnelles par approximations successives, Rev. Française Informat. Recherche Opérationnelle 4 (1970), 154-158 (French).

[10] S. Matsushita and W. Takahashi, A strong convergence theorem for relatively nonexpansive mappings in a Banach space, to appear.

[11] J.-J. Moreau, Un cas de convergence des itérées d'une contraction d'un espace hilbertien, C. R. Acad. Sci. Paris Sér. A-B 286 (1978), no. 3, A143-A144 (French).

[12] S. Reich, Constructive techniques for accretive and monotone operators, Applied Nonlinear Analysis (Proc. Third Internat. Conf., Univ. Texas, Arlington, Tex., 1978), Academic Press, New York, 1979, pp. 335-345.

[13] Weak convergence theorems for nonexpansive mappings in Banach spaces, J. Math. Anal. Appl. 67 (1979), no. 2, 274-276.

[14]_A weak convergence theorem for the alternating method with Bregman distances, Theory and Applications of Nonlinear Operators of Accretive and Monotone Type (A. G. Kartsatos, ed.), Lecture Notes in Pure and Appl. Math., vol. 178, Marcel Dekker, New York, 1996, pp. 313-318.

[15] R. T. Rockafellar, On the maximality of sums of nonlinear monotone operators, Trans. Amer. Math. Soc. 149 (1970), 75-88.

[16] Monotone operators and the proximal point algorithm, SIAM J. Control Optim. 14 (1976), no. 5, 877-898.

[17] W. Takahashi, Convex Analysis and Approximation Fixed Points, Yokohama Publishers, Yokohama, 2000 (Japanese).

[18] Nonlinear Functional Analysis. Fixed Point Theory and Its Applications, Yokohama Publishers, Yokohama, 2000.

[19] H. K. Xu, Inequalities in Banach spaces with applications, Nonlinear Anal. 16 (1991), no. 12, $1127-1138$.

Shin-ya Matsushita: Department of Mathematical and Computing Sciences, Tokyo Institute of Technology, Oh-Okayama, Meguro-ku, Tokyo 152-8552, Japan

E-mail address: shinya2@is.titech.ac.jp

Wataru Takahashi: Department of Mathematical and Computing Sciences, Tokyo Institute of Technology, Oh-Okayama, Meguro-ku, Tokyo 152-8552, Japan

E-mail address: wataru@is.titech.ac.jp 